\title{
Secretion of extracellular hsp90a via exosomes increases cancer cell motility: a role for plasminogen activation
}

\author{
Jessica McCready ${ }^{1}$, Jessica D Sims ${ }^{1}$, Doug Chan² and Daniel G Jay*1
}

\begin{abstract}
Background: Metastasis is a multi-step process that is responsible for the majority of deaths in cancer patients. Current treatments are not effective in targeting metastasis. The molecular chaperone hsp90a is secreted from invasive cancer cells and activates MMP-2 to enhance invasiveness, required for the first step in metastasis.

Methods: We analyzed the morphology and motility of invasive cancer cells that were treated with exogenous exosomes in the presence or absence of hsp90a. We performed mass spectrometry and immunoprecipitation to identify plasminogen as a potential client protein of extracellular hsp90a. Plasmin activation assays and migration assays were performed to test if plasminogen is activated by extracellular hsp90a and has a role in migration.

Results: We found that hsp90a is secreted in exosomes in invasive cancer cells and it contributes to their invasive nature. We identified a novel interaction between hsp90a and tissue plasminogen activator that together with annexin II, also found in exosomes, activates plasmin. Extracellular hsp90a promotes plasmin activation as well as increases plasmin dependent cell motility.

Conclusions: Our data indicate that hsp90a is released by invasive cancer cells via exosomes and implicates hsp90a in activating plasmin, a second protease that acts in cancer cell invasion.
\end{abstract}

\section{Background}

Approximately $90 \%$ of cancer deaths are not from the primary tumor but due to metastasis to distant sites [1]. Current treatments do not target metastatic disease. Towards developing anti-metastasis drugs, a functional proteomic screen was performed to identify surface proteins required for tumor cell invasion, the first step in metastasis [2]. One of the proteins identified was the molecular chaperone heat shock protein $90 \alpha$ (hsp90 $\alpha$ ) [2]. Intracellular hsp90 $\alpha$ aids in the folding, assembly-disassembly and activation of a variety of client proteins including kinases, steroid hormone receptors and transcription factors [3]. We discovered that extracellular hsp90 $\alpha$ acts in tumor cell invasion through its activation of the pro-invasive protein matrix metalloproteinase-2 (MMP-2). Since the publication of this study, additional

* Correspondence: daniel.jay@tufts.edu

1 Department of Physiology, Tufts University, 136 Harrison Ave, Boston, MA 02111, USA

Full list of author information is available at the end of the article reports in the literature have demonstrated the importance of extracellular hsp $90 \alpha$ in both physiological and pathological states. Extracellular hsp $90 \alpha$ is required for both dermal fibroblast [4] and neuronal motility [5] as well as for melanoma migration [6], invasion and metastasis [7].

The secretion method of extracellular hsp90 $\alpha$ from invasive cancer cells has not been fully elucidated. Hsp90 $\alpha$ has been found in exosomes in immune and other physiologically normal cell types [8-11] and suggested to be in exosomes in diabetic cells [12]. Exosomes are small vesicles, approximately $30-100 \mathrm{nM}$ in diameter, that are part of the endocytic pathway. They are secreted as intact vesicles that form within multivesicular bodies (MVB) and are released from cells when the membrane of the MVB fuses with the plasma membrane. Exosomes function in the immune system and in acellular communication [13]. Recent reports indicate that exosomes contribute to the aggressive nature of gliomas by transferring 
the mutated EGFRvIII receptor between cells [14]. The presence of hsp90 $\alpha$ in exosomes of other cells types and the observation that exosomes contribute to glioma aggressiveness suggested to us that hsp90 $\alpha$ in exosomes might contribute to cancer invasiveness.

In this study, we demonstrate that hsp90 $\alpha$ is secreted from invasive cancer cells via exosomes and increases cancer cell migration. We show that extracellular hsp $90 \alpha$ is necessary for the activation of a second extracellular protease, plasmin, and that fibrosarcoma cell movement is dependent on this activation.

\section{Methods}

\section{Cell culture}

A172, HT-1080, and MDA-MB231 cells were obtained from ATCC and maintained in DMEM supplemented with $10 \%$ FBS, $1 \%$ NEAA, and $1 \%$ P/S. SUM159 cells were a kind gift from Charlotte Kuperwasser and were maintained in Hams F12 media supplemented with 5\% FBS, 5 $\mu \mathrm{g} / \mathrm{mL}$ insulin, $10 \mathrm{ng} / \mathrm{mL}$ EGF and $1 \% \mathrm{P} / \mathrm{S}$. All cells were grown in a $37^{\circ} \mathrm{C}$ incubator with $7.5 \% \mathrm{CO}_{2}$.

\section{Quantitative Real time PCR}

Total RNA was extracted from MDA-MB231 breast cancer cell lines with TRIzol (Invitrogen, California) and $2 \mu \mathrm{g}$ of RNA was reverse transcribed into cDNA with Superscript III (Invitrogen) following the instructions supplied by the supplier. Real time PCR was performed at the Tufts Univesity Center for Neuroscience Research using the Stratagene real time cycler. Primer sequences were as follows: HSP90AA1-1 forward 5'-GGCAGAGGCTGATAAG-AACG-3' and reverse 5'CCCAGACCAAGT TTGATCATCC-3'; HSP90AA1-2 forward 5'-CATCTGATGGTGTCTGGATCC-3' and reverse 5'-AATGGCTGCAGATCCTTGTAG-3'. Samples were analyzed using the 2- $\Delta \Delta \mathrm{CT}$ method (29) with GAPDH as the reference.

\section{Brefeldin A Treatment}

MDA-MB231 cells were treated with $10 \mu \mathrm{g} / \mathrm{mL}$ Brefeldin A (BFA), (Sigma, Missouri) or vehicle control for 16 hours. Conditioned media was collected, concentrated and subjected to SDS-PAGE followed by a Western blot probed with MMP-2 antibody (EMD Biosciences, New Jersey), anti-hsp90 $\alpha$ or $\beta$-actin antibody (Sigma, Missouri). $\beta$-actin protein should be absent in conditioned media samples isolated from intact, alive cells.

\section{RNAi Treatment}

MDA-MB231 cells were transfected with either control siRNA (non-targeting) or $100 \mathrm{nM}$ siRNA directed against the HSP90AA1-2 (sense 5'-GTTAACTGGTACCAAGAAA-dTdT-3') isoform using Oligofectamine (Invitrogen). RNA was extracted as indicated above and the results are graphed as percentage knockdown setting the control at $100 \%$.

\section{Exosome isolation}

Exosomes were isolated from A172, HT-1080, MDAMB231, and SUM159 cells as previously described [8]. Briefly, $5 \times 10^{6}$ cells were plated in 10\% DMEM and allowed to settle overnight. Cells were then washed with HBSS and re-fed with serum free media or serum free media containing $15 \mathrm{nM}$ dimethyl amiloride (Sigma). Media was collected 48 hours after the addition of serum free DMEM and spun at $300 \times \mathrm{g}$ to collect any cellular debris. This media was then filtered with a $0.2 \mu \mathrm{M}$ filter and spun for 1 hour at $110,000 \times \mathrm{g}$. The pellet was washed with PBS and spun for 1 hour at $110,000 \times \mathrm{g}$. One $\mu \mathrm{g}$ of protein was subjected to Western Blot probed for hsp90 $\alpha$ (Assay Designs, Michigan). Samples were also probed with an anti-Annexin II antibody (BD Biosciences, California) and Flotillin (Cell Signaling Technology, Massachusetts) as positive controls and vATPase subunit B (Molecular Probes, California) as a negative control.

\section{Immunostaining}

$1 \times 10^{4}$ MDA-MB231 cells were plated into an 8-well chamber slide and treated with exosomes isolated from MDA-MB231 cells or $0.5 \mu \mathrm{g}$ recombinant hsp90 $\alpha$ (Assay Designs) for 16 hours. Cells were fixed in $\mathrm{PBS} / 4 \%$ paraformaldehyde $/ 4 \%$ sucrose, permeabilized in $0.1 \%$ TritonX-100/PBS, blocked in 1\%BSA/PBS and stained with Alexa546-labeled phalloidin (Invitrogen, CA) for $30 \mathrm{~min}$ utes to visualize F-actin.

\section{Cell shape analysis}

The cell shape and area of MDA-MB231 cells were measured and calculated with OpenLab software (Improvision). Cell shape was defined using the equation $(4 \times$ cell area)/cell perimeter ${ }^{2}$, where greater than 1 indicates a perfect circle and values less than 1 indicate a more irregular shape.

\section{Wound healing assay}

$1 \times 10^{5}$ SUM159 breast cancer cells or A172 glioma cells were plated in an 8 well chamber slide. Cells were wounded by scratching a sterile yellow pipette tip lengthwise along the chamber. The cells were washed twice with $1 \times$ PBS and serum free media was placed in each well with either the vehicle control PBS, $0.5 \mu \mathrm{g}$ recombinant hsp90 $\alpha$ protein, $1 \mu \mathrm{g}$ exosomes isolated from SUM159 cells, or $1 \mu \mathrm{g}$ exosomes isolated from SUM159 cells plus $40 \mu \mathrm{g} / \mathrm{mL}$ anti-hsp90 antibody (SPS-771, Assay Designs). Pictures were taken immediately after cell wounding (0 hours) and 16 hours after cell wounding. Wound width was calculated using OpenLab software and is represented as $\mu \mathrm{m}$ between the cells at 16 hours for each treatment. 


\section{Immunoprecipitation/Mass spectrometry}

$4 \times 10^{6}$ MDA-MB231 breast cancer cells were plated in a $150 \mathrm{~mm}$ tissue culture dish and allowed to settle for 24 hours. Cells were then refed with serum free media and incubated for 48 hours at $37^{\circ} \mathrm{C}$. Conditioned media was concentrated by centrifugation (Millipore, MA) and a protein assay was performed (BioRad, CA). $1 \mathrm{mg}$ of protein was pre-cleared with protein A beads after which 1 ug of hsp90 $\alpha$ antibody (Assay Designs) was added to the samples. Samples were washed with RIPA B buffer (50 $\mathrm{mM}$ Tris, $150 \mathrm{mM} \mathrm{NaCl}, 0.5 \%$ NP40, 0.25\% DOC) boiled, subjected to SDS PAGE, stained with Coomassie Blue and removed from the gel for mass spectrometry analysis. The excised gel bands were analyzed by mass spectrometry as previously described [15]. MS results were verified using antibodies for hsp90 $\alpha$ and tPA (Abcam, MA).

\section{Plasminogen activation assay}

Plasminogen activation assays were performed as previously described [16]. Briefly, HT-1080 fibrosarcoma cells were plated in 10\% DMEM and refed with serum free media 24 hours after plating. DMSO, $0.5 \mu \mathrm{M}$ [Glu] plasminogen (American Diagnostica Inc, CT), or $40 \mu \mathrm{g} / \mathrm{mL}$ DMAG-N-oxide (a gift from Len Neckers) were added for five hours at $37^{\circ} \mathrm{C}$. DMAG-N-oxide was used in this experiment because the large amount of antibody required for this experiment precluded its use. It has been previously characterized as an inhibitor of extracellular hsp90 $\alpha$ [17]. Conditioned media was concentrated (Millipore) and a protein assay was performed (Bio-Rad). $25 \mu \mathrm{g}$ of each sample was loaded into a $0.1 \%$ gelatin zymogram. The zymogram was washed twice for two hours each in wash buffer (50 mM Tris- $\mathrm{HCl}, 150 \mathrm{mM} \mathrm{NaCl}, 2.5 \%$ (v/v) Triton X-100, pH 7.4), three times for 5 minutes each in water and then incubated in wash buffer for 12 hours at $37^{\circ} \mathrm{C}$. The zymogram was stained with $0.5 \%$ coomassie, destained, and densitometry was performed to determine the plasminogen activation levels of each condition.

\section{Migration assay}

HT-1080 fibrosarcoma cells were plated in 10\% DMEM. 48 hours after plating the cells were labeled with CMTMR (Invitrogen) and $1 \times 10^{5}$ labeled cells were plated into a 24-well Fluoroblok plate (BD Biosciences, CA). Cells were treated with either $40 \mu \mathrm{g} / \mathrm{mL}$ rabbit IgG, $40 \mu \mathrm{g} / \mathrm{mL}$ anti-hsp90 $\alpha$ (SPS-771, Assay Designs), or 0.5 $\mu \mathrm{g}$ plasmin (Molecular Innovations, MI). Cells were allowed to migrate for 24 hours after which the number of cells that migrated to the bottom chamber were photographed and counted.

\section{Results}

Hsp90a is secreted via exosomes in invasive cancer cells

While the importance of extracellular hsp90 $\alpha$ for tumor cell migration, invasion and metastasis has been recog- nized, the mechanism that invasive tumor cells use to secrete hsp $90 \alpha$ is still unclear. In response to our work (2), it was hypothesized that alternative splicing of hsp $90 \alpha$ created multiple isoforms and that perhaps there was a mainly intracellular hsp90 $\alpha$ isoform and a mainly extracellular isoform of hsp90 $\alpha$ (18). This was of interest because one could then target drugs to the extracellular isoform without interfering with the important intracellular functions of hsp90 $\alpha$. We verified that the two hypothesized Hsp90 $\alpha$ isoforms existed in MDA-231 cells and determined the relative amounts using Real Time PCR (Figure 1a). We found that the primary isoform present in MDA-231 cells was the classical ten-exon isoform (AA1-1). The other isoform, AA1-2, with two additional exons, was present in only very small quantities. Even though there was only a small amount of the second isoform present, we hypothesized that this isoform could be targeted outside of the cell since the amount of Hsp90 $\alpha$ inside of the cell is much greater than the amount outside of the cell. In order to test if Hsp90 $\alpha$ was indeed being exported via a signal sequence in the two extra exons, we treated MDA-231 cells with Brefeldin A, a compound that inhibits the export of proteins through the canonical pathway. We collected the conditioned media from the cells, subjected it to SDS-PAGE and blotted for Hsp90 $\alpha$ (Figure 1b). Hsp90 $\alpha$ was detected outside

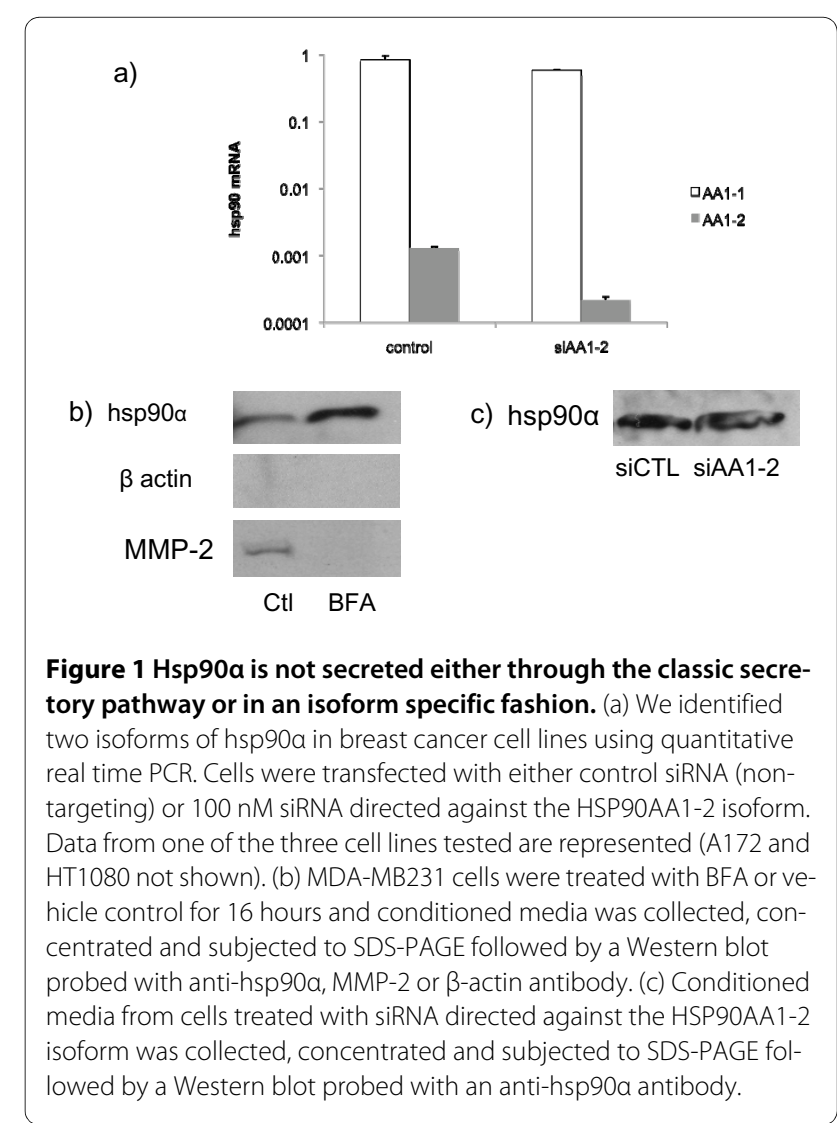


of the cell after BFA treatment in both cell lines indicating that it is not exported through the canonical signal sequence pathway. In fact, the extracellular hsp $90 \alpha$ protein levels were markedly increased by Brefeldin A, probably in response to cellular stress caused by this inhibitor. As a positive control we probed for MMP-2, a protein known to be exported via a signal sequence, and we found that its secretion was markedly inhibited by BFA (Figure 1b).

Even though Hsp90 $\alpha$ is not exported via a signal sequence, it may still be exported in an isoform specific manner. In order to test this we used siRNA to knock down the AA1-2 isoform. We obtained approximately $80 \%$ knock down of this isoform in the cells (Figure 1a) but did not see any reduction in the amount of Hsp90 $\alpha$ outside of the cell (Figure 1c). These results indicate that hsp $90 \alpha$ is not secreted by the classical secretory pathway or in an isoform specific manner. This led us to explore other non-classical secretory pathways such as exosomes. We isolated exosomes from MDA-MB231 cells and showed by immunoblot that they contain hsp $90 \alpha$ (Figure $2 \mathrm{a})$. To test the generality of this, we isolated exosomes from three other invasive tumor cell lines from different lineages, A172 glioblastoma cells, HT-1080 fibrosarcoma cells, and SUM159 breast cancer cells. The exosomes isolated from these cells also contain hsp90 $\alpha$ (Figure 2a). Purity of our exosome preparations was verified by the presence of annexin II and flotillin, two markers for exosomes, as well as by the absence of the large B subunit of the v-ATPase (Figure 2b), which is found in lysosomes

a)

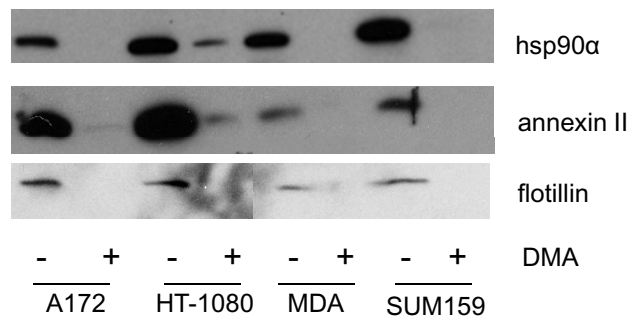

b)

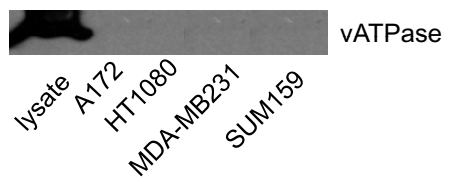

Figure $2 \mathrm{Hsp} 90 \mathrm{a}$ is present in the exosomes of four cancer cell lines. Western blot analysis of exosomes isolated from invasive cancer cell lines treated with vehicle (-) or DMA (+), which blocks the exosome pathway. Proteins were resolved by electrophoresis on 10\% SDS-PAGE and transferred to a nitrocellulose membrane. Western blot was performed using hsp90a, annexin II, flotillin, and vATPase antibodies. Representative images taken from three independent experiments are shown. and plasma membrane but not in exosomes [18]. When cells were treated with dimethyl amiloride (DMA), which blocks the exosome pathway[19], isolated exosome preparations from all cell lines tested showed marked reduction in hsp90 $\alpha$ as well as exosome markers (Figure 2a). Both hsp $90 \alpha$ and annexin II were degraded in a protease protection assay suggesting that they are easily accessible to extracellular proteins (data not shown). Therefore, hsp $90 \alpha$ in exosomes is accessible to activate secreted extracellular proteins such as MMP-2 [2].

\section{Exosomes induce a change in morphology of breast cancer} cells

Recent reports indicate that cancer cells secrete exosomes containing annexins and major histocompatibility complex proteins, which are normally associated with exosomes but also contain proteins involved in cell adhesion and motility such as integrins and fibronectin [2022]. These reports, along with our previously published data regarding the role of extracellular hsp90 $\alpha$ and tumor cell motility, suggest that exosomes containing hsp $90 \alpha$ released by invasive cancer cells could increase tumor cell motility. To test this hypothesis we isolated exosomes from the breast cancer cell line MDA-MB231, added them to the media of MDA-MB231 cells plated the previous day and stained the cells with phalloidin to analyze the morphology of the cells in the presence of exosomes (Figure 3a). Cells exposed to the control vehicle, PBS, display normal morphology, whereas cells exposed to exosomes for 16 hours show a more polarized shape associated with a motile phenotype. We also exposed MDA-MB231 cells to recombinant hsp90 $\alpha$ protein to determine if the effect we see with the exogenous exosomes is due in part to the presence of hsp90 $\alpha$ in the exosomes. Hsp90 $\alpha$ treated cells are more polarized than control-treated cells but less polarized than the cells treated with exosomes. To quantitate these changes, we measured both the cell shape and cell area using the OpenLab software program (Figure 3b). A motile cell can have a more linear cell shape as well as a large cellular area. We measured the perimeter of cells treated with either PBS, recombinant hsp $90 \alpha$ protein or exosomes. The data are represented in a Chang plot that depicts the shape of cells in which a perfect circle is greater than 1 and a perfect line is 0 . Cells treated with PBS have the most circular shape (average $=0.79 \pm 0.02$ ), cells treated with exosomes have the least circular shape (average = $0.49 \pm 0.02$ ) and cells treated with recombinant hsp $90 \alpha$ show intermediate values between those seen for control and exosome treated cells (average $=0.61 \pm 0.03$ ). Cells treated with either the recombinant hsp $90 \alpha$ or exosomes are statistically significantly different in shape when compared to the control treated cells $(\mathrm{p}<0.001)$. Exosome treatment also significantly increases the cell area when compared to cells treated with PBS or recombinant 
a)
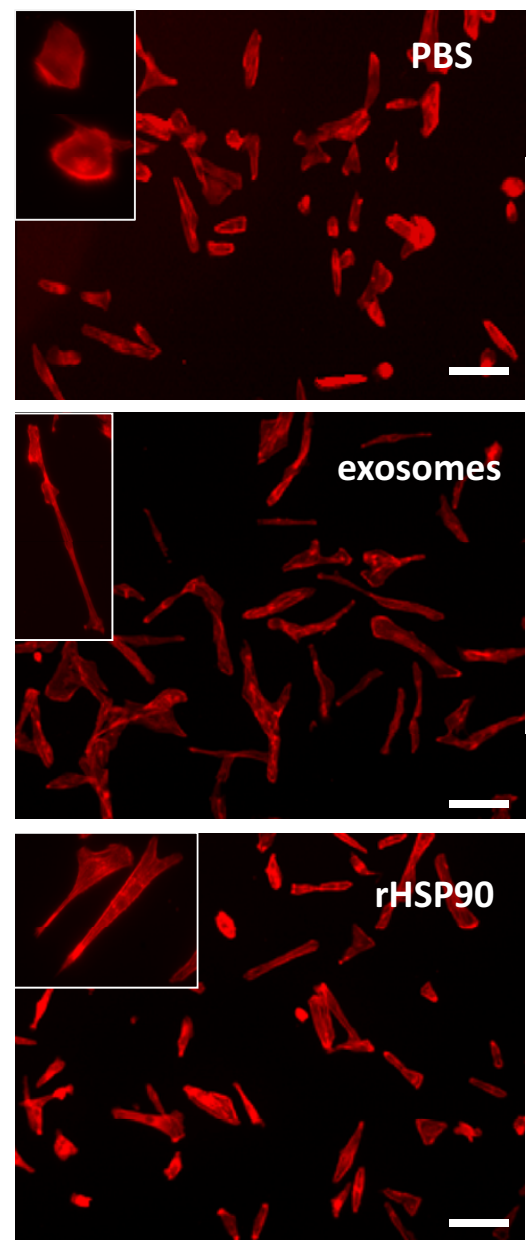

b)

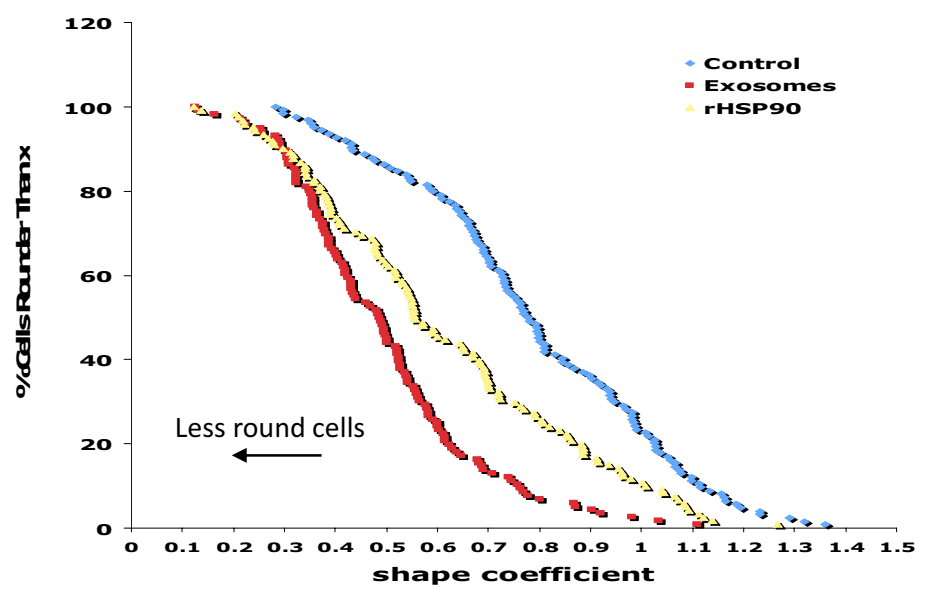

c)

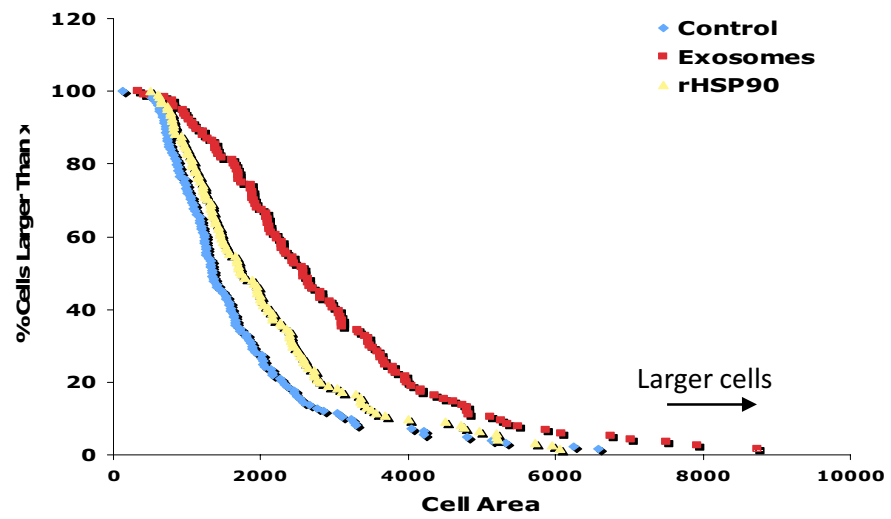

Figure 3 Exosomes alter the morphology of invasive breast cancer cells. Immunofluorescence and cell morphological analysis of MDA-MB231 cells. (a) Cells were treated with PBS (top panel), exosomes isolated from MDA-MB231 cells (middle panel) or recombinant hsp90a protein (bottom panel) and stained with phalloidin to visualize F actin. Images taken at 10x magnification from three independent experiments. Scale bar $=100 \mu \mathrm{M}$. Inset: Representative images at 20x magnification. (b) Chang plot showing cell shape measurements. Cell shape is defined using the equation (4x cell area)/cell perimeter ${ }^{2}$, where greater than 1 indicates a perfect circle and smaller values indicate a more irregular shape. Cell shape was calculated using OpenLab software. $n=130$. (c) Cell area analysis of MDA-MB231 cells treated with exogenous exosomes or recombinant hsp90a. Cell area was calculated using OpenLab software. $n=130$.

hsp90 $\alpha$ (Figure 3c). Cell area increased from an average of $1137 \pm 121 \mu^{2}$ to an average of $1398 \pm 138 \mu \mathrm{m}^{2}$ with the addition of recombinant hsp $90 \alpha(\mathrm{p}<0.02)$ and further increased to an average of $1914 \pm 167 \mu \mathrm{m}^{2}$ with the addition of exosomes $(\mathrm{p}<0.001)$. Taken together these results indicate that exogenous exosomes cause changes in both cell shape and cell area, consistent with motile behavior. We performed this experiment with A172 glioma cells (data not shown), however since they display a polarized morphology under normal growing conditions, the addition of exosomes did not affect their morphology.

\section{Exosomes increase cancer cell motility}

To determine if the changes in cell morphology translate to an increase in cell motility we performed wound healing assays. Since MDA-MB231 cells are not well suited to the wound healing assay we used two highly motile and invasive cell lines originating from different tumors: SUM159 breast cancer cells (Figure 4a) and A172 glioma cells (Figure 4b). We imaged the wound immediately after wounding the cells (0 hours) and added either recombinant hsp $90 \alpha$, exosomes, or exosomes plus a function inhibiting hsp90 $\alpha$ antibody [4]. We captured a second set of images 16 hours later. SUM159 control cells moved significantly less than cells treated with either $0.5 \mu \mathrm{g}$ recombinant hsp90 $\alpha(\mathrm{p}<0.05)$ or $1 \mu \mathrm{g}$ exosomes ( $\mathrm{p}<$ 0.01 ). The effect of exosomes on SUM159 cells was reduced by the hsp $90 \alpha$ function inhibiting antibody suggesting that hsp90 $\alpha$ is required for an exosome-dependent increase in cell movement. Control cells moved significantly less than cells treated with either recombi- 


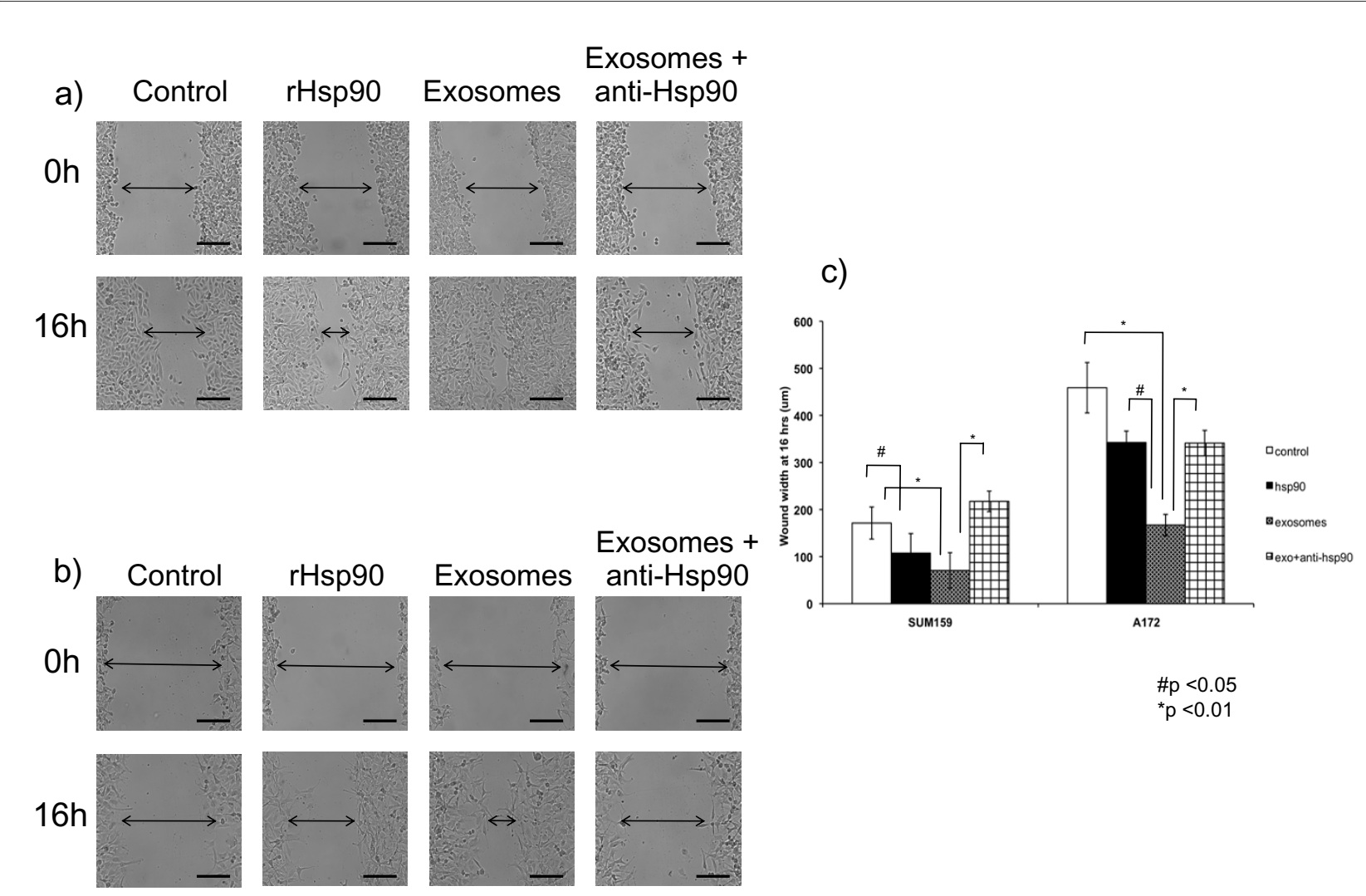

Figure 4 Exosomes increase hsp90a dependent glioma and breast cancer cell movement. (a) SUM159 breast cancer cells were wounded and images captured immediately and 16 hours after wounding. Cells were treated with either PBS, recombinant hsp90a protein, exosomes isolated from SUM159 cells or exosomes isolated from SUM159 cells plus anti-hsp90a antibody. Representative images from three independent experiments are shown for each time point and condition. Scale bar $=150 \mu \mathrm{M}$ (b) A172 glioma cells were wounded and images captured immediately or 16 hours after wounding. Cells were treated with either PBS, recombinant hsp90a protein, exosomes isolated from A172 cells or exosomes isolated from A172 cells plus anti-hsp90a antibody. Representative images from three independent experiments are shown for each time point and condition. Scale bar $=150 \mu \mathrm{M}$ (c) Graphical representation of the wound healing assay with SUM159 and A172 cancer cell lines. The addition of both recombinant hsp90a protein and anti-hsp90a antibody results in a significant decrease in wound closure at 16 hours $(p<0.05, p<0.01$ respectively, two tailed test).

nant hsp90 $\alpha(\mathrm{p}<0.05)$ or exosomes $(\mathrm{p}<0.01)$. A172 cells acted similarly to SUM159 cells in the wound healing assay. Data from the wound healing assay is represented graphically in Figure 4c.

\section{Extracellular hsp90a immunoprecipitates with tissue plasminogen activator}

Our findings established that the addition of exogenous exosomes changed both the morphology and motility of cancer cells. We had previously implicated hsp90 $\alpha$ in the activation of MMP-2, but it is possible that hsp $90 \alpha$ secreted via exosomes could activate other extracellular proteins. We hypothesized that extracellular hsp $90 \alpha$ was interacting with other extracellular proteins to increase cancer cell motility. To identify proteins that associate with hsp90 $\alpha$ we performed immunoprecipitation with conditioned media from MDA-MB231 cells followed by mass spectrometry (Figure 5a). We discovered ten proteins bound to extracellular hsp90 $\alpha$. The identified pro- teins are in their precursor form suggesting that they might be potential clients for this chaperone. We focused on one that could be linked to both exosomes and cell motility: tissue plasminogen activator protein (tPA). Annexin II, a protein secreted via exosomes, binds to both TPA and plasminogen and has been shown to associate with hsp90 $\alpha$ [23]. This binding initiates conversion of plasminogen to the protease plasmin [24]. We verified the mass spectrometry result with co-immunoprecipitation of Hsp90 $\alpha$ and tPA in conditioned media from HT-1080 fibrosarcoma cells (Figure 5b), the cell line for which we originally discovered the interaction of extracellular hsp90 $\alpha$ and MMP-2, and MDA-MB231 cells (data not shown). tPA and hsp90 $\alpha$ weakly interact in both MDAMB231 and HT-1080 cells perhaps because client and chaperone proteins interact transiently [25]. This raises the possibility that IPA is a novel client protein for extracellular hsp $90 \alpha$. 
\#2310-2310 RT:11.15-11.15 NL: 4.39E4

a)

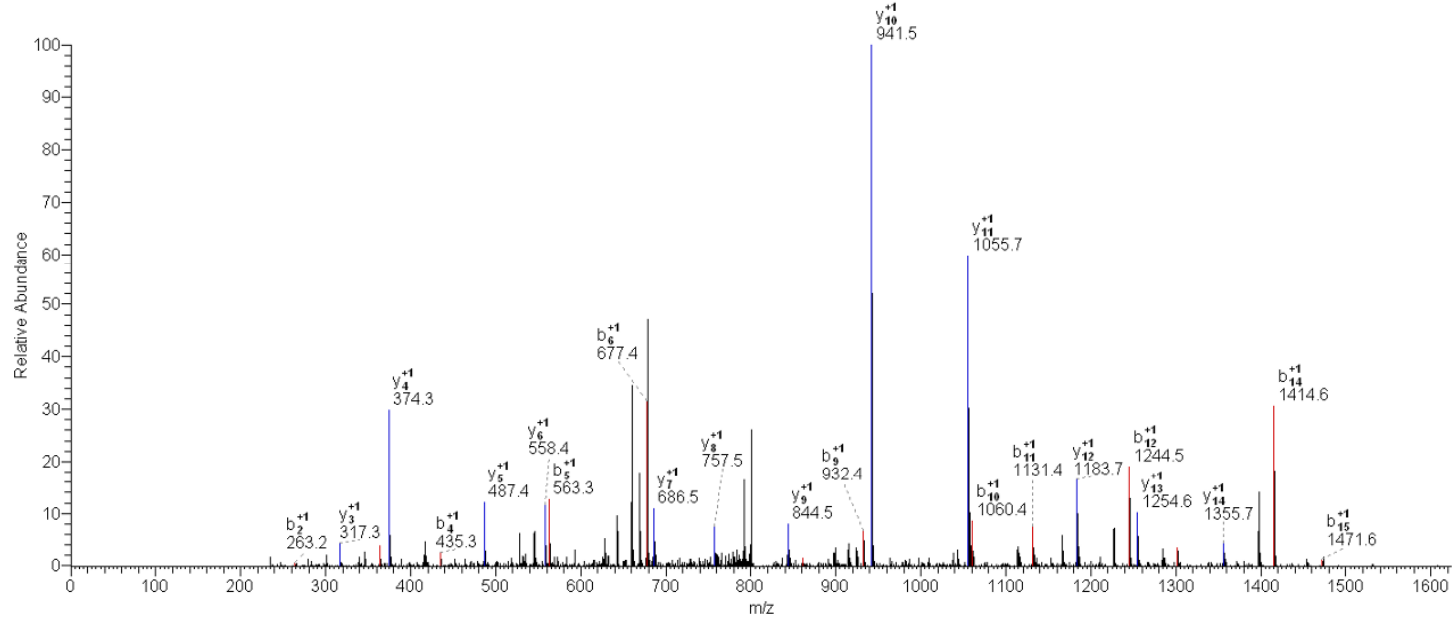

b)

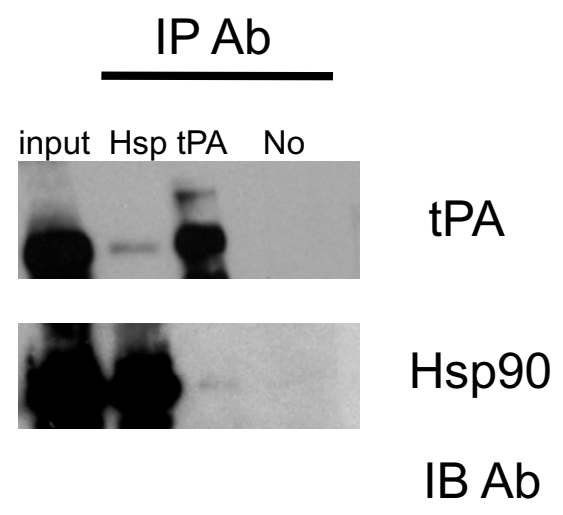

Figure 5 Extracellular hsp90a co-immunoprecipitates with tissue plasminogen activator. (a) MS/MS spectrum of the peptide

(248VYTAQNPSAQALGLGK263) that was used to identify tPA as a HSP90a interacting protein. (b) Immunoprecipitation of hsp90a from the conditioned media of HT-1080 fibrosarcoma cells. Hsp90a was immunoprecipitated from the media of HT-1080 cells and resolved on 10\% SDS-PAGE. Western blot analysis was performed using anti-hsp90a or anti-tPA antibodies. The input lane contains approximately $1 / 100^{\text {th }}$ of the total media collected. "IP Ab" indicates the antibody used during immunoprecipitation (hsp90a, tPA or no antibody as the negative control). "IB Ab" indicates which antibody was used the probe the immunoblot. Representative images from three independent experiments are shown.

\section{Hsp90a aids in the conversion of plasminogen to plasmin}

Previous data from our lab indicated that extracellular hsp $90 \alpha$ increases cancer cell invasion by assisting in the activation of MMP-2 [2]. To test if extracellular hsp90 $\alpha$ can promote the activation of other extracellular proteins involved in cancer cell motility we assessed whether the activation of plasmin through its association with tPA requires extracellular hsp $90 \alpha$. We performed plasmin activation assays with HT-1080 fibrosarcoma cells in the presence or absence of an inhibitor of extracellular hsp90 $\alpha$ (Figure 6a). We used DMAG-N-oxide, an impermeable form of the hsp90 $\alpha$ inhibitor geldanamycin [17] to determine if extracellular hsp90 $\alpha$ activates plasmin. The addition of DMAG-N-oxide resulted in a $32 \%$ decrease in activated plasmin when compared to cells that were treated with vehicle alone (Figure $6 \mathrm{~b}, \mathrm{p}<0.02$ ). These findings indicate that extracellular hsp $90 \alpha$ is involved in the conversion of plasminogen to plasmin.

Inhibition of extracellular hsp90a decreases tumor cell migration

While extracellular hsp90 $\alpha$ can activate plasmin, it was not known if this activation contributes to increased tumor cell motility. We performed transwell migration assays using a function-inhibiting antibody against hsp90 $\alpha$ (Figure 7). This antibody will only inhibit the extracellular hsp90 $\alpha$ because antibodies are membrane impermeant. HT-1080 fibrosarcoma cells treated with the hsp $90 \alpha$ antibody migrated $37 \%$ less than control treated cells $(\mathrm{p}<0.01)$. Interestingly, the addition of plasmin alone did not increase cell motility. Perhaps the cells are 


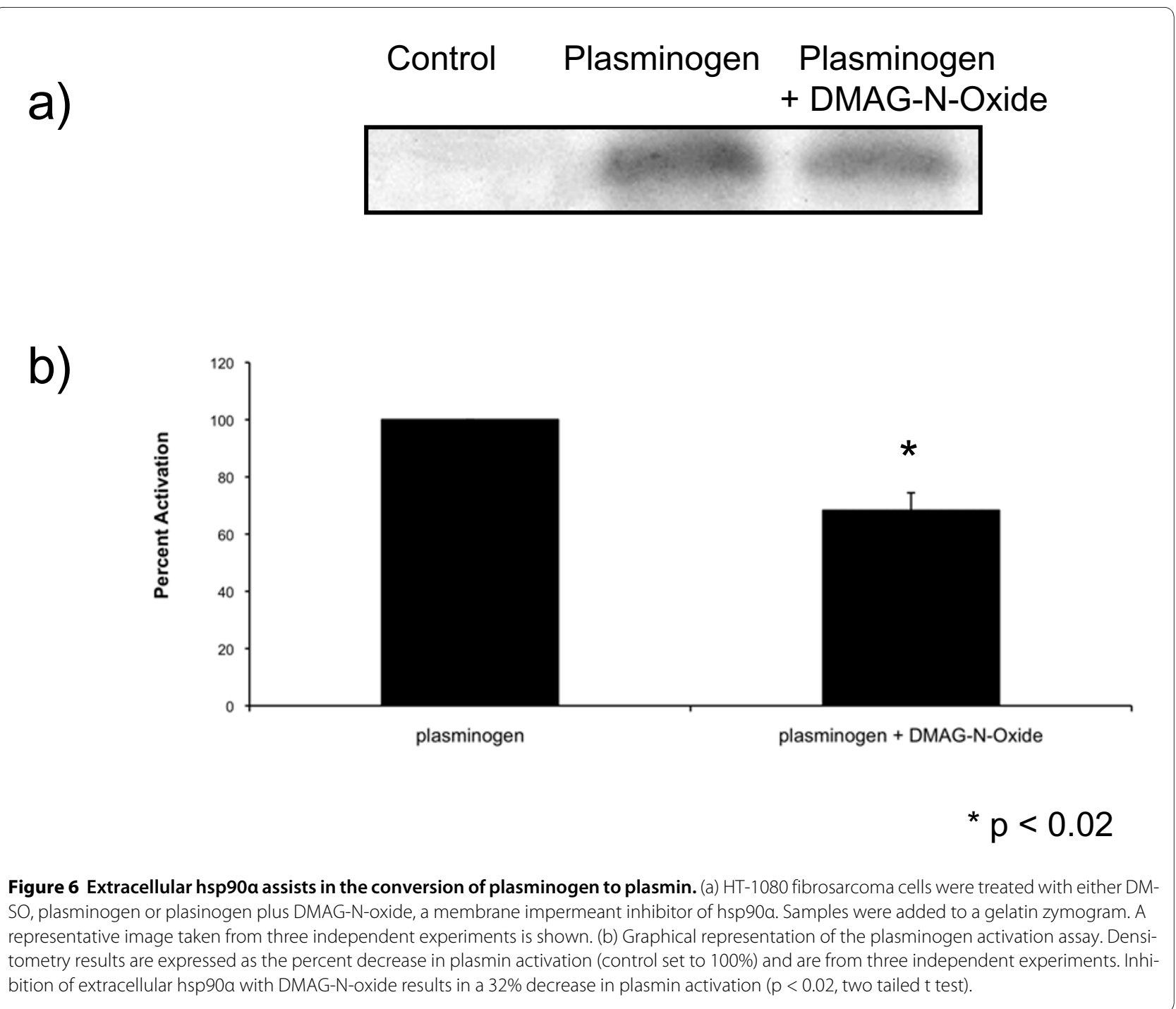

already saturated with active plasmin such that additional plasmin would not affect migration. Normal migration was recovered when we added activated plasmin to the cells treated with hsp90 $\alpha$ antibody. Together, these findings indicate that hsp $90 \alpha$ can activate plasmin and this activity stimulates cell motility.

\section{Discussion}

In this study we present evidence that extracellular hsp90 $\alpha$, secreted via exosomes, activates a novel client protein and increases tumor cell motility. Previously published work from our lab and others indicate that extracellular hsp90 $\alpha$ contributes to the activation of both MMP-2 [2] and HER-2 [26], two proteins involved in cancer metastasis. We now present data indicating that extracellular hsp $90 \alpha$ is necessary for the activation of a second protease, plasmin, also involved in tumor metastasis [27]. Inhibiting extracellular hsp90 $\alpha$ in vivo inhibits both wound healing [4] and tumor invasion [17]. Our current findings suggest however that other exosomal proteins may also contribute to these processes. Hsp $90 \alpha$ protein alone does not elicit as complete an effect on cell morphology or movement as the addition of exosomes (Figures 3 and 4). Hsp90 $\alpha$ binds to tPA and inhibiting hsp $90 \alpha$ decreased plasmin activation and cell migration. Therefore, we speculate that hsp90 $\alpha$ is part of an extracellular complex including annexin II, tPA and plasminogen that functions to increase cell movement. Annexin II is found in exosomes and has an established role in aggressive tumors and binds both tPA and plasminogen thereby enhancing the conversion of plasminogen to active plasmin [24]. Extracellular hsp90 $\alpha$ increases annexin II at the cell surface in rat aortic cells, leading to an increase in plasmin production in these cells [23]. Also, cell surface annexin II expression levels are increased in metastatic tumors and it interacts with multiple extracellular pro- 


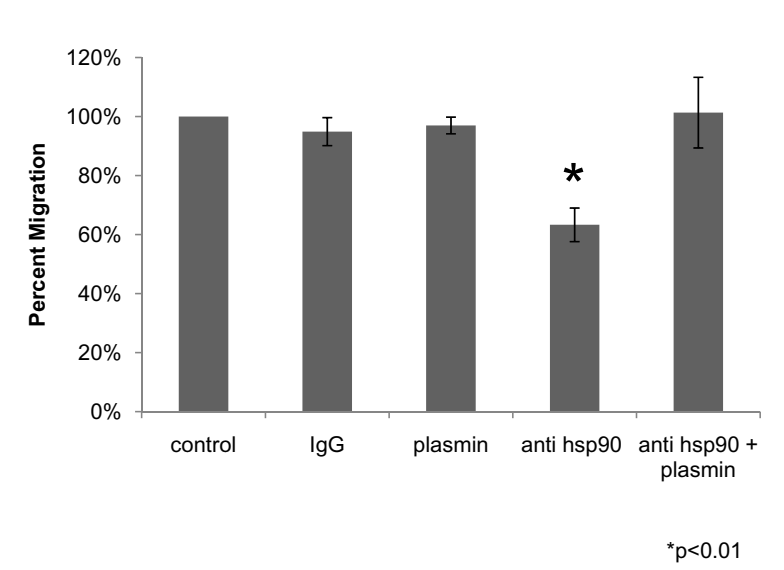

Figure 7 Inhibition of extracellular hsp90a inhibits cancer cell motility by preventing plasmin activation. $\mathrm{HT}-1080$ cells were treated with control, lgG, plasmin, anti-hsp90a antibody, or plasmin plus anti-hsp90a antibody. A transwell migration assay was performed and cells that migrated through the chamber were counted. Results are from four independent experiments and expressed as percentage decrease from control (100\%). Anti-hsp90a antibody results in a 37\% decrease in migration ( $p<0.01$, two tailed $t$ test).

teases that have been implicated in tumor progression [28]. Although plasmin is known for its role in cellular invasion it has not been well studied in migration, one component of the multi-step process of tumor invasion. Plasmin may be contributing to cell migration by contributing to the local remodelling of the extracellular matrix exposing cryptic cell attachment sites necessary for cellular migration, similar to that seen in smooth muscle cells during wound healing [29]. It is also possible that plasmin contributes to cell migration by interacting with currently unknown targets.

We suggest that exosome contents are released outside the tumor cell in close proximity to each other and other inactive extracellular pro-invasive proteins such as plasminogen. Once released from the exosomes, extracellular hsp90 $\alpha$ assists in the activation of pro-MMP2 as well as plasminogen. Beyond MMP-2 and plasmin it is possible that hsp $90 \alpha$ could activate other extracellular proteins as most of the proteins identified by mass spectrometry in this study were found in their inactive pro-forms. It is therefore interesting to speculate that extracellular hsp $90 \alpha$ could activate a cassette of proteins that function collectively in cancer cell migration. These proteins would act in concert to enhance breakdown and remodeling of the extracellular matrix and permit the tumor cell to invade its microenvironment. Thus, inhibition of extracellular hsp90 $\alpha$ could inhibit a growing number of proteins that are responsible for increased tumor cell movement making extracellular hsp90 $\alpha$ an attractive target for drug therapy to limit tumor invasion.

\section{Conclusions}

In summary, we have identified that exosomes increase cell motility. One mechanism for this increased motility is the activation of plasmin by extracellular hsp $90 \alpha$. The discovery of a second protease activated by extracellular hsp90 $\alpha$ suggests the possibility that the one role of extracellular hsp90 $\alpha$ in cancer cells is the activation of precursor proteins that contribute to cellular migration and invasion.

\section{Competing interests \\ JM, JDS, DC, DGJ: none declared.}

\section{Authors' contributions}

JM contributed to study design, data interpretation, carried out all of the experiments and drafted the manuscript. JDS contributed to experiments in Figure 1, to study design, data interpretation and helped revise the manuscript. DC carried out the mass spectrometry. DGJ contributed to study design, data interpretation and editing of the manuscript. All authors have read and approved the final manuscript.

\section{Acknowledgements}

The authors would like to thank J Dice, M Forgac, L Liscum, and T Bagci for their advice during the preparation of this manuscript. This work is supported by a grant from the NIH: R01 CA 116642 (DG Jay) and an NIH training grant T32 DK007542-21 (J McCready).

\section{Author Details}

1Department of Physiology, Tufts University, 136 Harrison Ave, Boston, MA 02111, USA and 2Protech Laboratory Inc, Houston, TX 77054, USA

Received: 8 October 2009 Accepted: 16 June 2010

Published: 16 June 2010

\section{References}

1. Rabbani SA, Mazar AP: Evaluating distant metastases in breast cancer: from biology to outcomes. Cancer Metastasis Rev 2007, 26(3-4):663-674.

2. Eustace BK, Sakurai T, Stewart JK, Yimlamai D, Unger C, Zehetmeier C, Lain B, Torella C, Henning SW, Beste G, et al:: Functional proteomic screens reveal an essential extracellular role for hsp90 alpha in cancer cell invasiveness. Nat Cell Biol 2004, 6(6):507-514.

3. Jackson SE, Queitsch C, Toft D: Hsp90: from structure to phenotype. Nat Struct Mol Biol 2004, 11(12):1152-1155.

4. Li W, Li Y, Guan S, Fan J, Cheng CF, Bright AM, Chinn C, Chen M, Woodley DT: Extracellular heat shock protein-90alpha: linking hypoxia to skin cell motility and wound healing. EMBO J 2007, 26(5):1221-1233.

5. Sidera K, Samiotaki M, Yfanti E, Panayotou G, Patsavoudi E: Involvement of cell surface HSP90 in cell migration reveals a novel role in the developing nervous system. J Biol Chem 2004, 279(44):45379-45388.

6. Becker B, Multhoff G, Farkas B, Wild PJ, Landthaler M, Stolz W, Vogt T: Induction of $\mathrm{Hsp} 90$ protein expression in malignant melanomas and melanoma metastases. Exp Dermatol 2004, 13(1):27-32.

7. Stellas D, Karameris A, Patsavoudi E: Monoclonal antibody 4C5 immunostains human melanomas and inhibits melanoma cell invasion and metastasis. Clin Cancer Res 2007, 13(6):1831-1838.

8. Thery C, Boussac M, Veron P, Ricciardi-Castagnoli P, Raposo G, Garin J, Amigorena S: Proteomic analysis of dendritic cell-derived exosomes: a secreted subcellular compartment distinct from apoptotic vesicles. J Immunol 2001, 166(12):7309-7318.

9. Pisitkun $T$, Shen RF, Knepper MA: Identification and proteomic profiling of exosomes in human urine. Proc Natl Acad Sci USA 2004, 101(36):13368-13373.

10. Clayton A, Turkes A, Navabi H, Mason MD, Tabi Z: Induction of heat shock proteins in B-cell exosomes. Journal of Cell Science 2005, 118(Pt 16):3631-3638.

11. Cheng CF, Fan J, Fedesco M, Guan S, Li Y, Bandyopadhyay B, Bright AM Yerushalmi D, Liang M, Chen M, et al:: Transforming growth factor alpha (TGFalpha)-stimulated secretion of HSP90alpha: using the receptor 
LRP-1/CD91 to promote human skin cell migration against a TGFbetarich environment during wound healing. Mol Cell Biol 2008, 28(10):3344-3358

12. Lei H, Venkatakrishnan A, Yu S, Kazlauskas A: Protein kinase A-dependent translocation of $\mathrm{Hsp} 90$ alpha impairs endothelial nitric-oxide synthase activity in high glucose and diabetes. J Biol Chem 2007 282(13):9364-9371.

13. Denzer K, Kleijmeer MJ, Heijnen HF, Stoorvogel W, Geuze HJ: Exosome: from internal vesicle of the multivesicular body to intercellular signaling device. Journal of Cell Science 2000, 113(Pt 19):3365-3374.

14. Al Nedawi K, Meehan B, Micallef J, Lhotak V, May L, Guha A, Rak J: Intercellular transfer of the oncogenic receptor EGFRvIll by microvesicles derived from tumour cells. Nat Cell Biol 2008, 10(5):619-624

15. Daquinag A, Fadri M, Jung SY, Qin J, Kunz J: The yeast PH domain proteins SIm 1 and SIm 2 are targets of sphingolipid signaling during the response to heat stress. Molecular and Cellular Biology 2007, 27(2):633-650.

16. Choi KS, Fogg DK, Yoon CS, Waisman DM: p11 regulates extracellular plasmin production and invasiveness of HT1080 fibrosarcoma cells. FASEB J 2003, 17(2):235-246.

17. Tsutsumi S, Scroggins B, Koga F, Lee MJ, Trepel J, Felts S, Carreras C, Neckers L: A small molecule cell-impermeant $\mathrm{Hsp} 90$ antagonist inhibits tumor cell motility and invasion. Oncogene 2008, 27(17):2478-2487.

18. Thery C, Zitvogel L, Amigorena S: Exosomes: composition, biogenesis and function. Nat Rev Immunol 2002, 2(8):569-579.

19. Savina A, Furlan M, Vidal M, Colombo Ml: Exosome release is regulated by a calcium-dependent mechanism in K562 cells. J Biol Chem 2003 278(22):20083-20090.

20. Hegmans JP, Bard MP, Hemmes A, Luider TM, Kleijmeer MJ, Prins JB, Zitvogel L, Burgers SA, Hoogsteden HC, Lambrecht BN: Proteomic analysis of exosomes secreted by human mesothelioma cells. Am J Pathol 2004, 164(5):1807-1815.

21. Mears R, Craven RA, Hanrahan S, Totty N, Upton C, Young SL, Patel P, Selby PJ, Banks RE: Proteomic analysis of melanoma-derived exosomes by two-dimensional polyacrylamide gel electrophoresis and mass spectrometry. Proteomics 2004, 4(12):4019-4031.

22. Yu X, Harris SL, Levine AJ: The regulation of exosome secretion: a novel function of the p53 protein. Cancer Research 2006, 66(9):4795-4801.

23. Lei $\mathrm{H}$, Romeo G, Kazlauskas A: Heat shock protein 90alpha-dependent translocation of annexin II to the surface of endothelial cells modulates plasmin activity in the diabetic rat aorta. Circ Res 2004, 94(7):902-909.

24. Hajjar KA, Krishnan S: Annexin II: a mediator of the plasmin/ plasminogen activator system. Trends Cardiovasc Med 1999, 9(5):128-138.

25. Whitesell L, Lindquist SL: HSP90 and the chaperoning of cancer. Nat Rev Cancer 2005, 5(10):761-772.

26. Sidera K, Gaitanou M, Stellas D, Matsas R, Patsavoudi E: A critical role for HSP90 in cancer cell invasion involves interaction with the extracellular domain of HER-2. J Biol Chem 2008, 283(4):2031-2041.

27. Sharma MR, Koltowski L, Ownbey RT, Tuszynski GP, Sharma MC: Angiogenesis-associated protein annexin II in breast cancer: Selective expression in invasive breast cancer and contribution to tumor invasion and progression. Exp Mol Pathol 2006, 81(2):146-156.

28. Mai J, Waisman DM, Sloane BF: Cell surface complex of cathepsin B/ annexin II tetramer in malignant progression. Biochim Biophys Acta 2000, 1477(1-2):215-230.

29. Stefansson S, Lawrence DA: The serpin PAI-1 inhibits cell migration by blocking integrin alpha V beta 3 binding to vitronectin. Nature 1996, 383(6599):441-443.

Pre-publication history

The pre-publication history for this paper can be accessed here: http://www.biomedcentral.com/1471-2407/10/294/prepub

doi: 10.1186/1471-2407-10-294

Cite this article as: McCready et al., Secretion of extracellular hsp90? via exosomes increases cancer cell motility: a role for plasminogen activation BMC Cancer 2010, 10:294

\section{Submit your next manuscript to BioMed Central} and take full advantage of:

- Convenient online submission

- Thorough peer review

- No space constraints or color figure charges

- Immediate publication on acceptance

- Inclusion in PubMed, CAS, Scopus and Google Scholar

- Research which is freely available for redistribution 